\title{
CRT-D implantation: one procedure, two problems
}

\author{
Anna M. Engel, Jakub Machejek, Andrzej Ząbek, Artur Kozanecki, Jacek Lelakowski, Barbara Małecka
}

Department of Electrocardiology, John Paul II Hospital, Krakow, Poland

Adv Interv Cardiol 2016; 12, 3 (45): 282-284

DOI: $10.5114 /$ aic.2016.61657

A 70-year-old patient with chronic ischemic cardiomyopathy (NYHA III, left ventricle ejection fraction (EFLV) = $15 \%$, left bundle branch block (LBBB), percutaneous coronary intervention $(\mathrm{PCI})$ left anterior descending artery (LAD) in 2006 and right coronary artery (RCA) in 2007), after an episode of sustained VT, terminated pharmacologically, was qualified for a cardiac resynchronization therapy (CRT-D) procedure (class IA).

Reduction of heart failure was impossible despite optimal pharmacotherapy. The defibrillation lead was implanted in the right ventricle apex through the left cephalic venotomy, and the atrial lead was implanted in the right atrium appendage by a subclavian vein approach. Optimal atrial and ventricular pacing parameters were obtained.

The left ventricular (LV) lead was implanted through left subclavian venepuncture with difficulties of coronary sinus (CS) cannulation.

The cannulation of the vein running on the front wall of the heart - the marginal vein ( $M$. von Ludinghausen type III of CS anatomy - Figure 1) - was enabled by using an LV introducing set with small curvature (Medtronic Attain 6216A-MB2). Administered contrast visualized single tributaries and the CS trunk.

Cannulation of the CS was performed successfully based on the image of performed venography, followed by implantation of the LV lead in the wide lateral vein. Excellent pacing parameters were obtained in the distal location, although associated with diaphragm stimulation. More proximal reposition helped to gain acceptable LV stimulation parameters without any side effects. While the introducing sheath was removed, LV lead was dislocated with loss of effective stimulation.

Instead of the previous standard Medtronic guidewire, a hemodynamic guidewire (Abbott Hi-Torque Balance Heavyweight - Abbott-GW) was inserted into the lead, with the help of which an ineffective attempt of lead reposition was performed, using the over-the-wire technique.
Due to earlier difficulties with CS cannulation we decided to introduce a new sheath using Abbott-GW, the ending of which was already in the CS. To do this part of the procedure it was necessary to remove the lead and leave only the Abbott-GW. The sheath was inserted, the Abbott-GW removed, and the LV lead was implanted again in the lateral vein with optimal electrical parameters. The sheath and the standard guidewire were removed without lead reposition.

After the procedure was accomplished, reduction of the Abbott-GW's length $(25 \mathrm{~cm}$ ending with fragile structure) was noticed. In a chest X-ray the fragment of the guidewire was localized between the superior vena cava (SVC) and pulmonary trunk (PT) (Figure 2). The break of the Abbot-GW was probably the result of its vigorous removal from the wide sheath, and not as usual from the lumen of the pacemaker lead. Perhaps during abrupt removal of the sheath, a part of the guidewire was moved into the SVC: the firm ending was attached to the SVC, while the fragile one flowed with the blood stream towards the PT.

The AndraSnare AS-25 Set was used with venous access through the right ulnar vein. The guidewire fragment removal procedure was performed with the loop (Figure 3) which captured the guidewire and took it out from the venous system (Figure 4). The position of implanted leads was not changed. The patient was discharged in a good condition.

This case has been presented to show possible difficulties with the CRT-D implantation procedure: a variant of topography in the venous inflow and the complication of breakage of the hemodynamic guidewire.

As it is an uncommon complication of the CRT implantation procedure, there are few reports about leaving foreign bodies in the venous system, such as broken CVP catheters, metal guide wires, pacemaker electrodes, port-A fragments and vascular stents. Potential complications of a leaving foreign body include: septicemia,

\section{Corresponding author:}

Anna M. Engel MD, Department of Electrocardiology, John Paul II Hospital, 80 Prądnicka St, 31-202 Krakow, Poland, phone: +48 606796 185, e-mail: amkengel@gmail.com

Received: 14.05.2014, accepted: 11.02.2016. 

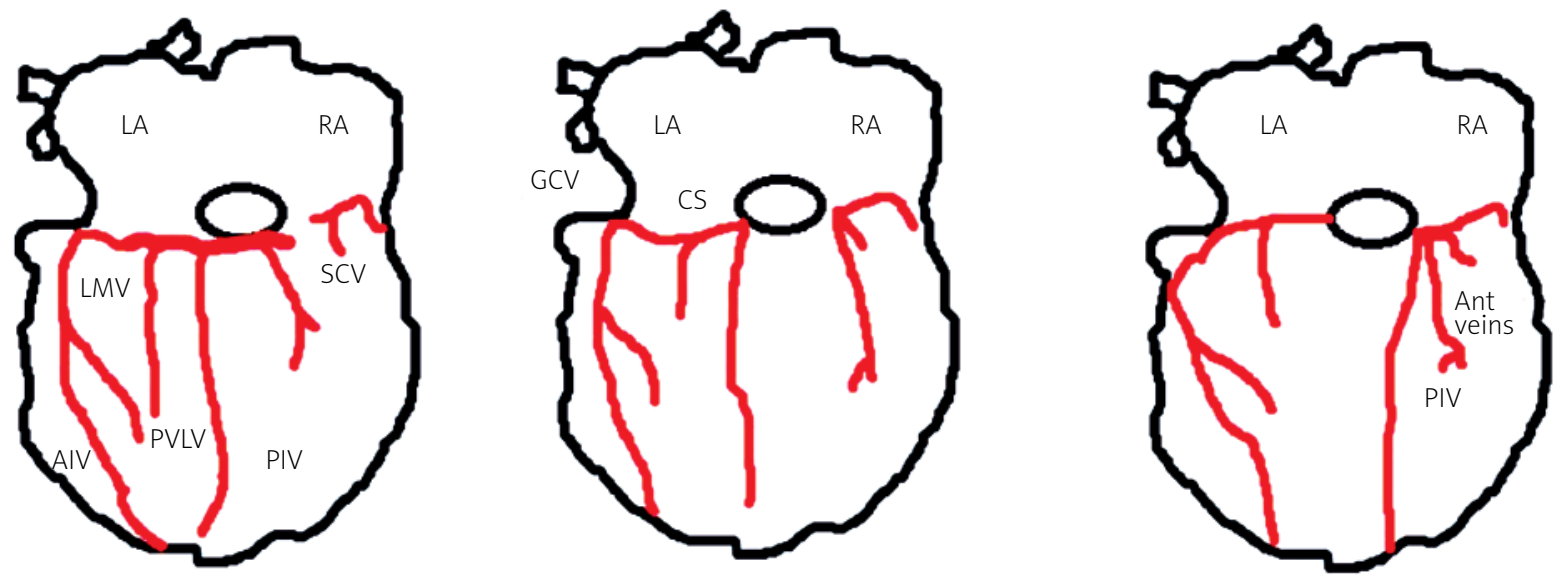

Figure 1. Types of CS (coronary sinus) anatomy according to M. von Ludinghausen

CS - coronary sinus, VM - vein of Marshall, GCV - great cardiac vein, AIV - anterior interventricular vein, RA - right atrium, LA - left atrium, PIV - posterior interventricular vein, PVLV - posterior vein of the left ventricle, $L M V$ - left marginal vein, SCV - small cardiac vein.
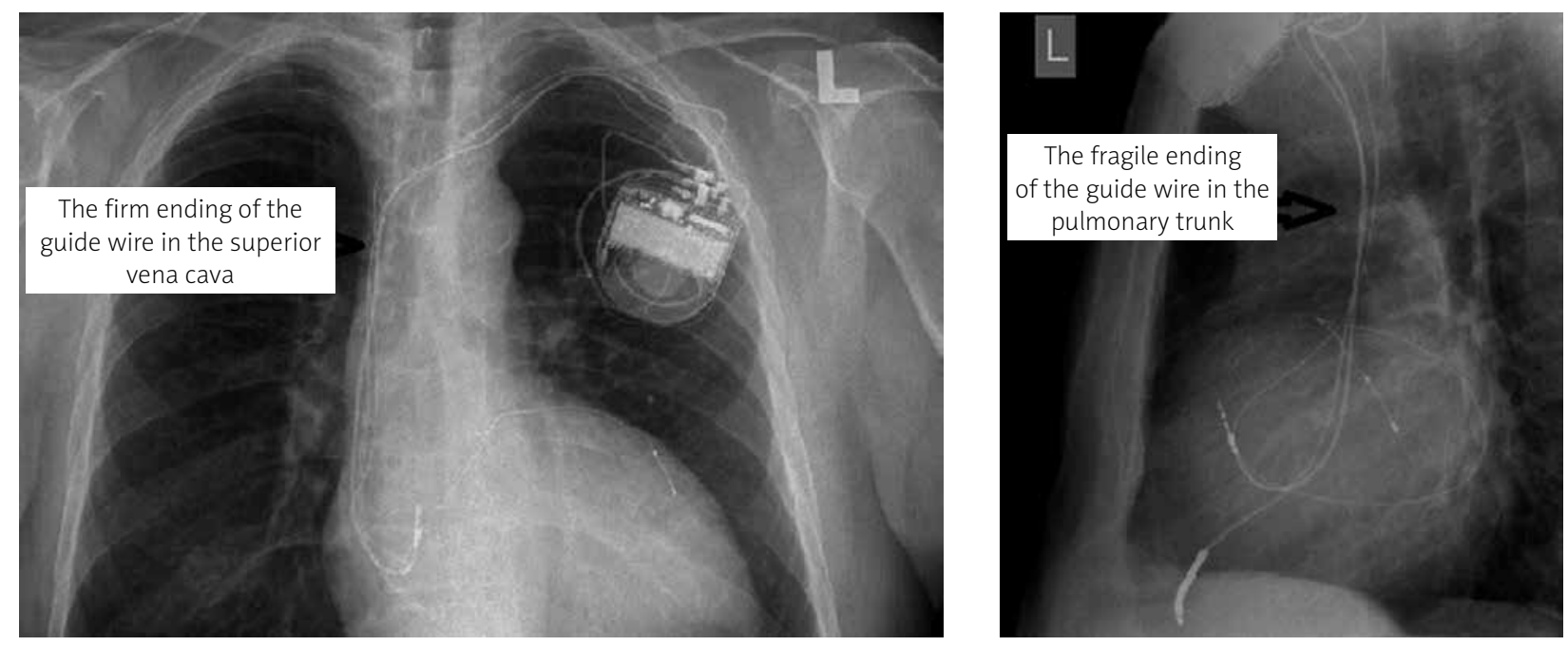

Figure 2. The chest X-ray with the broken hemodynamic guidewire
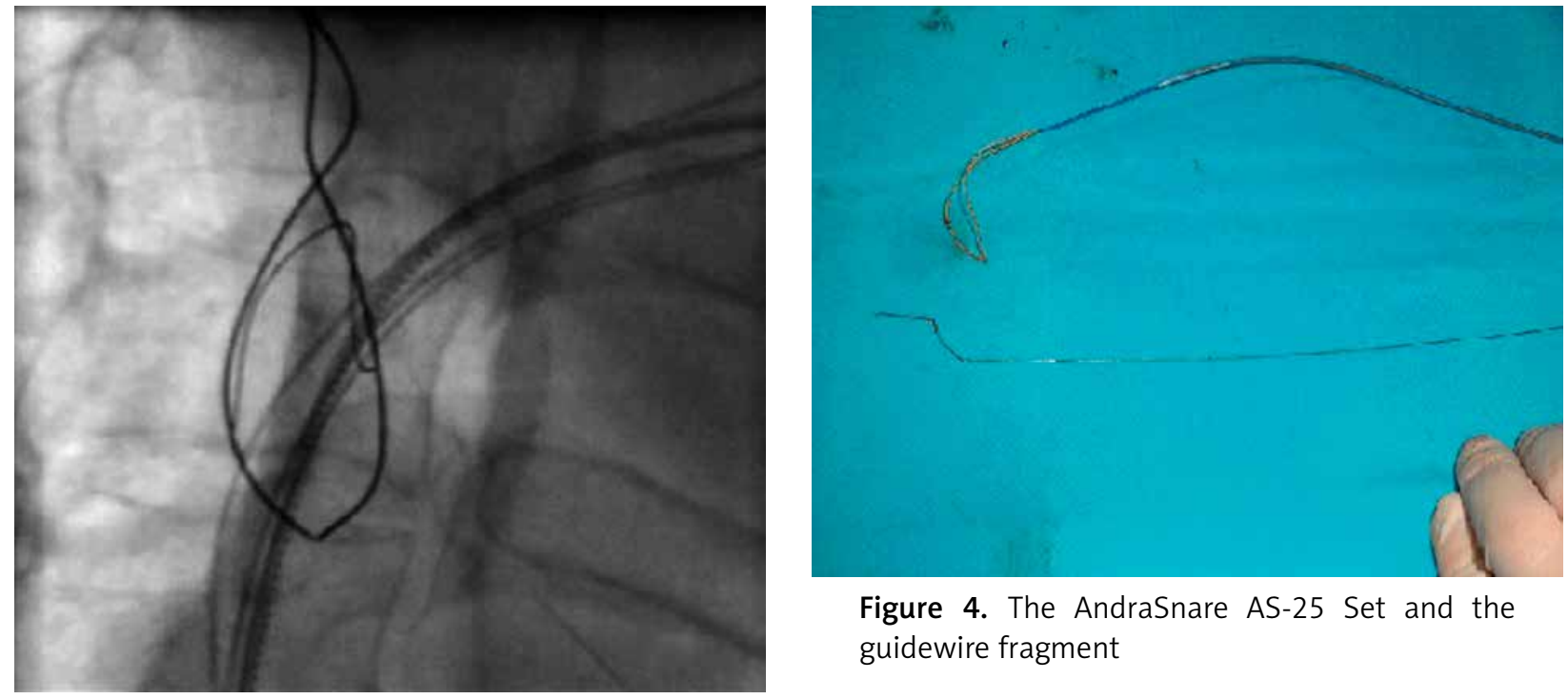

Figure 4. The AndraSnare AS-25 Set and the guidewire fragment

Figure 3. The guidewire fragment removal procedure, performed with the loop 
multiple pulmonary emboli, abscess formation, arrhythmia, perforation of great vessels or heart and death [1, 2]. In the present case expected complications could be: arrhythmia (as a part of the wire was already sliding on the wall of the right ventricle, gently leaning on the tricuspid valve), multiple pulmonary emboli (as the risk of formation of thrombi on the wire was increased) or damage to the pulmonary vessels.

The other uncommon complication is leaving a broken wire or stylet in the lumen of the lead. In time this leads to structural damage of the lead and its dysfunction. The removal after time (months/years) is a high risk procedure [3, 4].

There are also reports showing that leaving part of a lead in the cardiovascular system, in the right heart cavities or pulmonary artery may cause pulmonary embolism, and a lead left in the vessel leads to its occlusion [5].

\section{Conflict of interest}

The authors declare no conflict of interest.

\section{References}

1. Hu CC, Lin SC, Huang PH, et al. A lady with a broken hemodialis catheter fragment. Nephrol Dial Transplant 2002; 17: 1126-8.

2. Park SK, Yi IK, Lee JH, et al. Fracture of J-tipped guidewire during central venous catherization and its successful removal under fluoroscopic guidence - a case report. Korean J Anesthesiol 2012; 63: 457-60

3. Kay GN, Brinker JA, Kawanishi DT, et al. Risks of spontaneous injury and extraction of an active fixation pacemaker lead reportant of the Accufix Multicenter Clinical Study and Worldwide Registry. Circulation 1999; 100: 2344-52.

4. Williams TL, Bowdle TA, Winters BD, et al. Guidewires unintentionally retained during central venous catheterization. JAVA 2014; 19: 29-34.

5. Kutarski A, Małecka B, Ząbek A, Pietrucha R. Broken leads with proximal endings in the cardiovascular system: serious consequences and extraction difficulties. Cardiol J 2013; 20: 161-9. 